\title{
岩浆岩的地球化学成分记录了全球性氧化事件
}

\author{
侯增谦 \\ 中国地质科学院地质研究所, 北京 100037 \\ E-mail: houzq@nsfc.gov.cn
}

\section{Global oxygenation events recorded by igneous geochemistry}

\author{
Zengqian Hou \\ Institute of Geology, Chinese Academy of Geological Sciences, Beijing 100037, China \\ E-mail: houzq@nsfc.gov.cn \\ doi: 10.1360/TB-2019-0531
}

众所周知, 现在的大气中氧气含量占 $21 \%$. 但是在地 球早期, 大气中几乎没有氧气 ${ }^{[1]}$. 大气中的氧含量在地质 历史时期经历了两次大规模的升高事件, 分别是24 21亿 年前的“大氧化事件(Great Oxidation Event)” ${ }^{[2,3]}$ 和7.5 6亿 年前的“新元古代氧化事件”, ${ }^{[4,5]}$. 人们对这两次全球性氧 化事件精确发生时间的研究始于 20 世纪末 21 世纪初 ${ }^{[6]}$. 绝大部分研究是针对于沉积岩的地球化学特征而开展的, 这是因为沉积岩的元素含量、同位素组成等直接记录了大 气-海水中氧含量的变化. 而岩浆岩的地球化学成分虽然 也会受到大气氧含量的影响, 但岩浆作用过程较为复杂, 影响其成分的因素颇多, 难以用于精确限定氧化事件的发 生时间.

2019年9月, Proc Natl Acad Sci USA (PNAS)刊发了中 国科学院海洋研究所深海中心孙卫东课题组的研究论文 "Global atmospheric oxygen variations recorded by $\mathrm{Th} / \mathrm{U}$ systematics of igneous rocks" "[7], 采用全球弧岩浆岩的针元 素 $(T h)$ 和铀元素 $(\mathrm{U})$ 的比值 $(T h / U)$ 来指示前寒武纪大气氧含 量的变化, 以全新的手段和视角对全球性氧化事件的发生 时间进行了限定.

$\mathrm{Th}$ 和U是两种地球化学性质十分相近的元素, 它们在 岩浆作用过程中表现出相似的地球化学行为. Liu等人 ${ }^{[7]}$ 的统 计结果发现, 不同岩性的岩浆岩中平均 Th/U值是相对恒定 的, 亦即岩浆岩的 $\mathrm{Th} / \mathrm{U}$ 不会随着岩石中二氧化硅 $\left(\mathrm{SiO}_{2}\right)$ 含量 的改变而改变. 而自然界可以造成Th-U元素分离的主要原 因是地表氧化作用和海水蚀变作用. 针元素在自然界只有一 个稳定价态 $\mathrm{Th}^{4+}$, 而铀元素则有两个稳定价态 $\mathrm{U}^{4+}$ 和 $\mathrm{U}^{6+}$. 岩 浆岩中的 $\mathrm{Th}^{4+}$ 和 $\mathrm{U}^{4+}$ 均是不溶于水的, 但当岩浆岩出露于地 表, $\mathrm{U}^{4+}$ 被氧化为 $\mathrm{U}^{6+}$ 后则可以溶于水, 通常以 $\mathrm{UO}_{2}\left(\mathrm{CO}_{3}\right)_{3}{ }^{4-}$ 的 形式存在于河流和海水中 ${ }^{[8]}$. 针和铀元素的这种氧化还原性
差异导致现代海水中的铀元素含量远高于针元素含量, 从而 造成蚀变的洋壳具有低的 $\mathrm{Th} / \mathrm{U}$ 值. 蚀变洋壳在板块俯冲的 作用下, 将这种低 $T h / \mathrm{U}$ 特征带人到地幔楔中, 从而在俯冲带 上方形成了具有低 $T h / U$ 值的弧岩浆岩[7].

但是在大氧化事件以前 $(>24$ 亿年前), 大气中没有氧 气. 地表上的 $\mathrm{Th}$ 和 $\mathrm{U}$ 均以不溶于水的正四价 $\left(\mathrm{Th}^{4+}\right.$ 和 $\left.\mathrm{U}^{4+}\right)$ 的 形式存在, 地表上还存在大量碎屑状晶质铀矿 $\left(\mathrm{UO}_{2}\right)$, 当

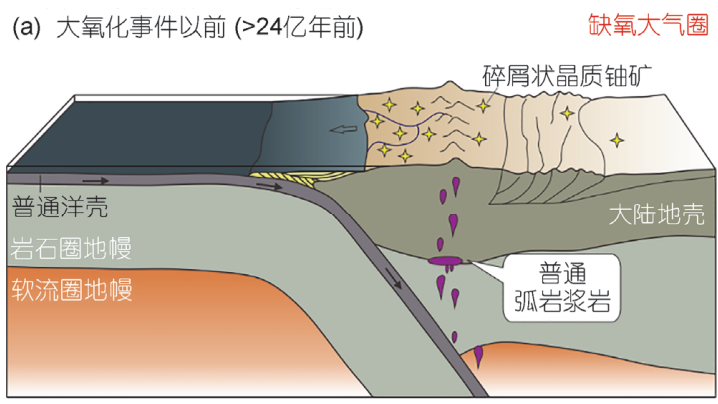

(b) 大氧化事件时 (24 20.5亿年前)

含氧大气圈

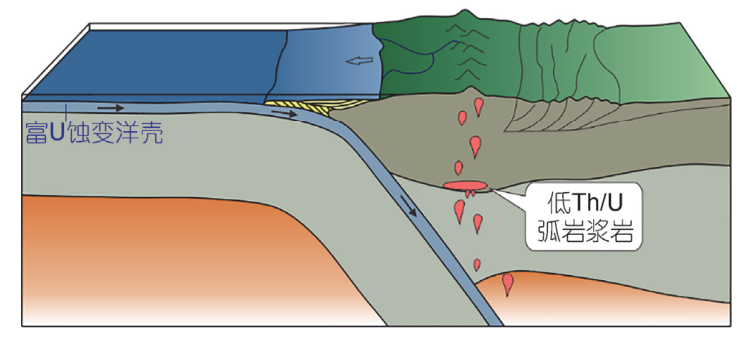

图 1 (网络版彩色)大氧化事件以前(a)和大氧化事件过程中(b)铀元 素的循环过程

Figure 1 (Color online) The uranium cycle before the Great Oxidation Event (a) and during the Great Oxidation Event (b) 
时形成的弧岩浆岩并不具有低 $T h / U$ 特征(图 1(a)). 当大氧 化事件发生时(24 20.5亿年前), 地表上的 $U^{4+}$ 被氧化为溶 于水的 $\mathrm{U}^{6+}$, 随地表河流最终汇人到海洋中, 此时形成的 弧岩浆岩则开始具有低 $T h / U$ 特征(图 1(b)). Liu等人 ${ }^{[7]}$ 根据 弧岩浆岩通常具有低 $\mathrm{Nb} / \mathrm{Th}$ 值、低 $T h$ 含量的特点, 从全球 岩浆岩数据库中篮选出所有中酸性弧岩浆岩, 以统计学方 法获得了从 30 亿年前到今天形成的弧岩浆岩的平均 $T h / U$ 值. 结果显示, 弧岩浆岩的 $\mathrm{Th} / \mathrm{U}$ 在地质历史时期的两次大 幅度降低刚好对应于两次全球性氧化事件的发生. 虽然弧 岩浆岩的 Th/U的两次降低时间分别发生于 23.5 亿年前和 7.5 亿年前, 略晚于前人根据南非Ongeluk大火成岩省的喷 发时代所定义的大氧化事件的时间 (24.2亿年前) ${ }^{[9]}$ 和根据 加拿大Wynniatt组具有 $\mathrm{Cr}$ 同位素异常的页岩年龄所限定的 新元古代氧化事件的时间 (8亿年前) ${ }^{[5]}$. 或许是因为弧岩浆 岩的成分受大气氧升高的影响会有一定的滞后. 但是大火
成岩省的喷发并不能代表全球性大气氧含量的升高, 页岩 的 $\mathrm{Cr}$ 同位素异常也可能是局部水体偏高的氧含量所致, 而 全球中酸性弧岩浆岩的平均 $\mathrm{Th} / \mathrm{U}$ 值的大幅度改变则更具 可信度.

值得指出的是, 弧岩浆岩的 Th/U可以同时显示两次 全球性氧化事件的发生. 大氧化事件的发生和结束的时间 是基于南非Ongeluk大火成岩省的喷发时代 ${ }^{[9]}$ 、沉积岩的硫 同位素 ${ }^{[2]}$ 和碳同位素组成的变化而限定的 ${ }^{[3]}$. 而新元古代 氧化事件的发生时间是通过沉积岩的 $\mathrm{Cr}$ 同位素组成 ${ }^{[5]} 、 \mathrm{Mo}$ 元素含量 ${ }^{[4]}$ 以及硫酸盐蒸发岩定年的结果 ${ }^{[10]}$ 而确定的. Liu 等人 ${ }^{[7]}$ 做出的全球弧岩浆岩 $\mathrm{Th} / \mathrm{U}$ 变化的统计结果, 是截至 目前所发表的众多研究成果中唯一可以同时限定两次氧 化事件时间的地球化学指标. 该研究的发表, 将有可能兴 起一个探索岩浆岩的地球化学成分与全球性氧化事件的 相关性的研究热潮.

\section{参考文献}

1 Lyons T W, Reinhard C T, Planavsky N J. The rise of oxygen in earth's early ocean and atmosphere. Nature, 2014, 506: 307-315

2 Bekker A, Holland H D, Wang P L, et al. Dating the rise of atmospheric oxygen. Nature, 2004, 427: 117-120

3 Planavsky N J, Bekker A, Hofmann A, et al. Sulfur record of rising and falling marine oxygen and sulfate levels during the Lomagundi event. Proc Natl Acad Sci USA, 2012, 109: 18300-18305

4 Sahoo S K, Planavsky N J, Kendall B, et al. Ocean oxygenation in the wake of the Marinoan glaciation. Nature, 2012, 489: 546-549

5 Planavsky N J, Reinhard C T, Wang X, et al. Low mid-proterozoic atmospheric oxygen levels and the delayed rise of animals. Science, 2014, 346: 635-638

6 Farquhar J, Savarino J, Jackson T L, et al. Evidence of atmospheric sulphur in the martian regolith from sulphur isotopes in meteorites. Nature, 2000, 404: 50-52

7 Liu H, Zartman R E, Ireland T R, et al. Global atmospheric oxygen variations recorded by Th/U systematics of igneous rocks. Proc Natl Acad Sci USA, 2019, 116: 18854-18859

8 Partin C A, Bekker A, Planavsky N J, et al. Large-scale fluctuations in precambrian atmospheric and oceanic oxygen levels from the record of U in shales. Earth Planet Sci Lett, 2013, 369: 284-293

9 Gumsley A P, Chamberlain K R, Bleeker W, et al. Timing and tempo of the Great Oxidation Event. Proc Natl Acad Sci USA, 2017, 114: 1811-1816

10 Turner E C, Bekker A. Thick sulfate evaporite accumulations marking a mid-Neoproterozoic oxygenation event (Ten Stone Formation, Northwest Territories, Canada). Geol Soc Am Bull, 2015, 128: 203-222 